\title{
Persistent genital arousal disorder: always look beyond the surface - a case report
}

\author{
Rita Sarabando $^{1 *}$, Natacha Sousa ${ }^{1}$, Ana C Borges ${ }^{1}$, Cristina Nogueira-Silva ${ }^{2}$
}

\begin{abstract}
${ }^{1}$ Department of Obstetrics and Gynaecology, Hospital de Braga, Braga, Portugal
${ }^{2}$ Department of Obstetrics and Gynaecology, Hospital de Braga, Braga; Life and Health Sciences Research Institute (ICVS), School of Medicine, University of Minho, Braga; ICVS/3B's - PT Government Associate Laboratory, Braga/ Guimarães, Portugal
\end{abstract}

Received: 15 February 2021

Accepted: 12 March 2021

\author{
*Correspondence: \\ Dr. Rita Sarabando, \\ E-mail: sarabandorita@gmail.com
}

Copyright: ( $)$ the author(s), publisher and licensee Medip Academy. This is an open-access article distributed under the terms of the Creative Commons Attribution Non-Commercial License, which permits unrestricted non-commercial use, distribution, and reproduction in any medium, provided the original work is properly cited.

\begin{abstract}
Persistent genital arousal disorder is a rare condition characterized by unwanted intrusive symptoms of sexual arousal without specific context. Their possible aetiologies and treatments are multiple and mostly based on case reports. We aim to do a comprehensive review of persistent genital arousal disorder and describe a case of a postmenopausal woman who developed this disease and, during the follow-up, was diagnosed with advanced endometrial cancer, reminding physicians to keep in mind the possibility of multiple diagnosis in the same patient, including malignancy. Although there is no description of this association in the literature, the possible aetiologies of persistent genital arousal disorder are diverse, and we sought this rare case should be disclosed.
\end{abstract}

Keywords: Persistent genital arousal disorder; Endometrial cancer; Malignancy

\section{INTRODUCTION}

Persistent genital arousal disorder (PGAD) is a rare medical condition characterized by symptoms of sexual arousal in the absence of perceived sexual stimulus. Its prevalence is still not known, but it is estimated to be about $1 \%$ of women. ${ }^{1}$ The social stigma and the women shame and fear to be misunderstood may contribute to the probable underestimation of this disorder prevalence. ${ }^{1,2}$ The symptoms include genital vasocongestion, increased sensitivity of the genitals and nipples, vaginal lubrication, genital tingling, throbbing, and contractions. Pelvic pain can also be present. ${ }^{2,3}$ In 2001, Leiblum and Nathan originally proposed five criteria for this condition: genital and clitoral arousal that persists for an extended period (hours, days, or months), symptoms do not resolve with ordinary orgasmic experience and might require multiple orgasms over hours or days to remit, genital arousal is unrelated to subjective feelings of sexual desire, persistent genital arousal can be triggered by sexual activity, nonsexual stimuli or by no apparent stimulus at all and a very persistent feelings of genital arousal is intrusive, unwanted and cause at least a moderate degree of distress. Sometimes distress is considered a separate, sixth criteria. ${ }^{4}$ Those criteria have been published by the International society for the study of women's sexual health (ISSWSH) in the consensus nomenclature for distressing sexual dysfunctions and are included in the recommendations made by the fourth international consensus on sexual medicine. ${ }^{5,6}$

The aetiology is still not completely known, but it can be related to central or peripheral neurological anomalies (peripheral nerve hypersensitivity or entrapment and neuronal hyperexcitability may be one of the main features of this disorder), Tarlov or sacral spinal cysts, pelvic congestion, pudendal neuralgia, use or withdrawal of medications such as anti-depressants, increased dietary soy intake, or psychological factors such as anxiety.,7 There seems to be also a relationship between PGAD and restless legs syndrome in a condition classified as restless genital syndrome, in which overactive bladder can also be 
present. $^{8}$ As the aetiology is still not completely understood, treatment is also a challenge. Nonpharmacological treatments include avoidance of tight clothing and of prolonged sitting, dietary modifications and counselling, also pelvic floor physiotherapy, nerve modulation or stimulation and surgical options (there are descriptions of removal of masses or spinal meningeal cysts, and embolization of pelvic veins). ${ }^{2,7,9}$ Pharmacological treatment options that may relieve PGAD symptoms include antiandrogens, anxiolytics, antidepressants, anticonvulsants, dopamine agonists and dopamine antagonists. ${ }^{2,7,9}$

This clinical case describes a diagnosis of PGAD and its follow-up, during which the patient was diagnosed with an endometrial cancer in an advanced stage. ${ }^{4}$

\section{CASE REPORT}

A 67 year old woman presented in a gynaecological appointment describing vaginal discharges that occurred with a sensation like sexual excitement for the last 3 months, more than 10 times a day and waking her up during the night. The patient denied urinary incontinence or urgency, and the sensation described was present in the absence of sexual desire or sexual activity. She denied ever having had sexual intercourse and referred menopause at the age of 51, with one year of hormone replacement therapy after that. She was asthmatic and was taking daily medication for high blood pressure and dyslipidaemia, with no other relevant illnesses, namely neurological or surgeries. She was not taking any other medications like anti-depressants or over-the-counter pills. On abdominal examination, the patient had no relevant findings, and on gynaecological examination (limited by the fact that the patient did not have coitarche) severe vulvovaginal atrophy was observed, probably related to genitourinary syndrome of menopause (SGUM), with no other relevant findings. The suspected diagnosis was PGAD. She denied other symptoms like paraesthesia and/or dysesthesia, genitopelvic, leg, or lower back pain, bladder symptoms or restless legs. In order to exclude organic pathology, a suprapubic ultrasound was performed and showed a uterus with $40 \times 60 \times 30 \mathrm{~mm}$, with $35 \mathrm{~mm}$ type 4 leiomyoma, without pelvic varices or other findings. According to the patient she already knew about this leiomyoma, which was present and stable for several years, without associated symptoms. Her hormonal blood analyses were within normal limits and urinalyses was normal too.

Treatment with cloxazolam $1 \mathrm{mg}$ per day was started, as well as local oestrogen and vaginal moisturizer. Her symptoms progressively improved with very much sporadic episodes after several months of treatment. Patient had an annual gynaecological appointment and denied any other symptom. Three years later she presented with postmenopausal genital bleeding and lower abdominal pain, and she was immediately observed. Gynaecological exam with one finger revealed some blood in vagina, without palpable masses. Suprapubic ultrasound showed a uterus with $85 \times 75 \times 65 \mathrm{~mm}$ with lobulated contours, heterogeneous texture and multiple myometrial intramural masses compatible with leiomyomas type 4, the biggest of them with $37 \times 29 \mathrm{~mm}$ (Figure 1). Endometrial cavity measure was $4.86 \mathrm{~mm}$ (Figure 2 ). There were no adnexal lesions and no free liquid in the abdominal cavity. She was recommended to do a hysteroscopy in which endometrial biopsies were done and showed endometrial serous carcinoma. In the thoracoabdominopelvic computed tomography done for staging, the uterus was multinodular with lobulated contours and the endometrium was thickened (Figures 3A and 3B). Some lymphadenopathies were seen at peri-aortocaval locations and around iliac vessels, with no other image findings of spread malignancy. She was referred to an oncologic gynaecological service. Hysterectomy with salpingooophorectomy, aortic and pelvic lymphadenectomy were performed, and complete resection was achieved (R0). The pathological examination confirmed the endometrial serous carcinoma with invasion of more than half of the myometrium, lymphovascular invasion, and metastases to pelvic and para-aortic lymph nodes (FIGO IIIC2). ${ }^{10}$ Patient is currently under adjuvant chemotherapy, and adjuvant radiotherapy is also planned. Patient is stable and asymptomatic at six months after surgery.

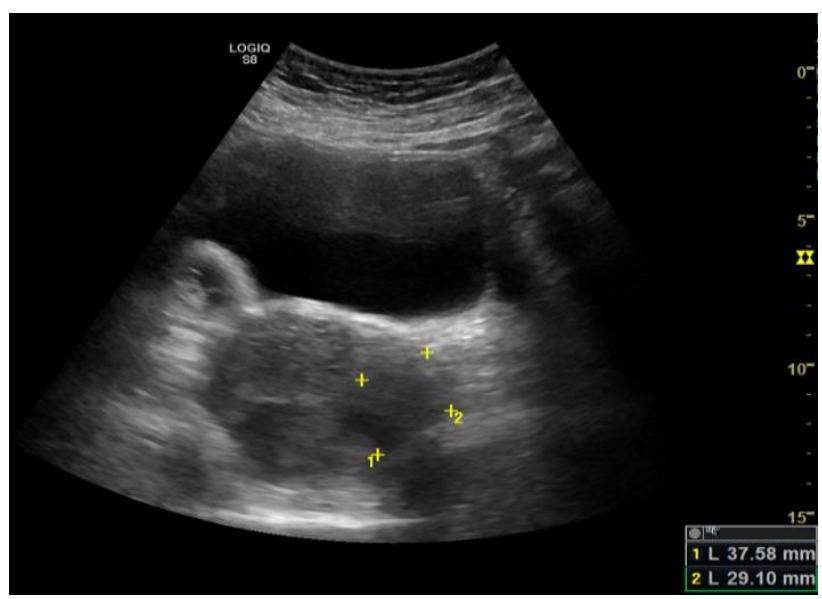

Figure 1: Suprapubic ultrasound - uterine leiomyoma.

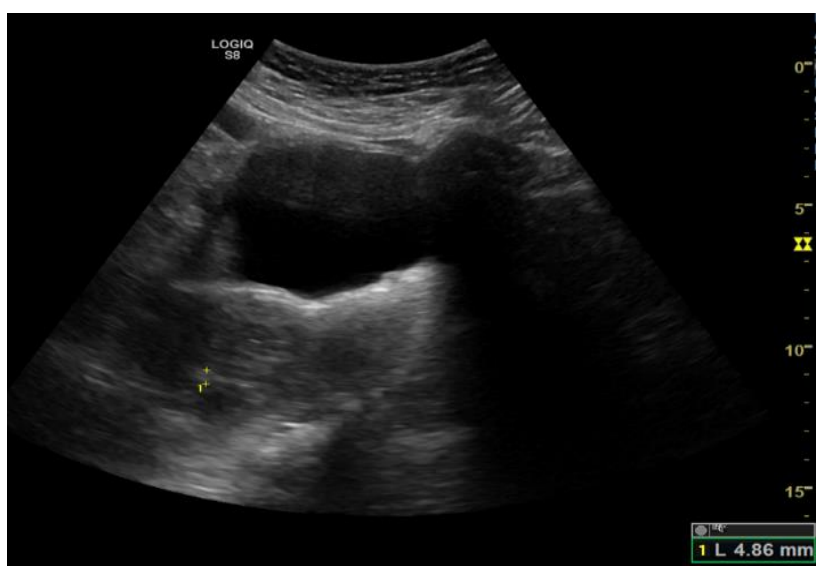

Figure 2: Suprapubic ultrasound - endometrial cavity. 


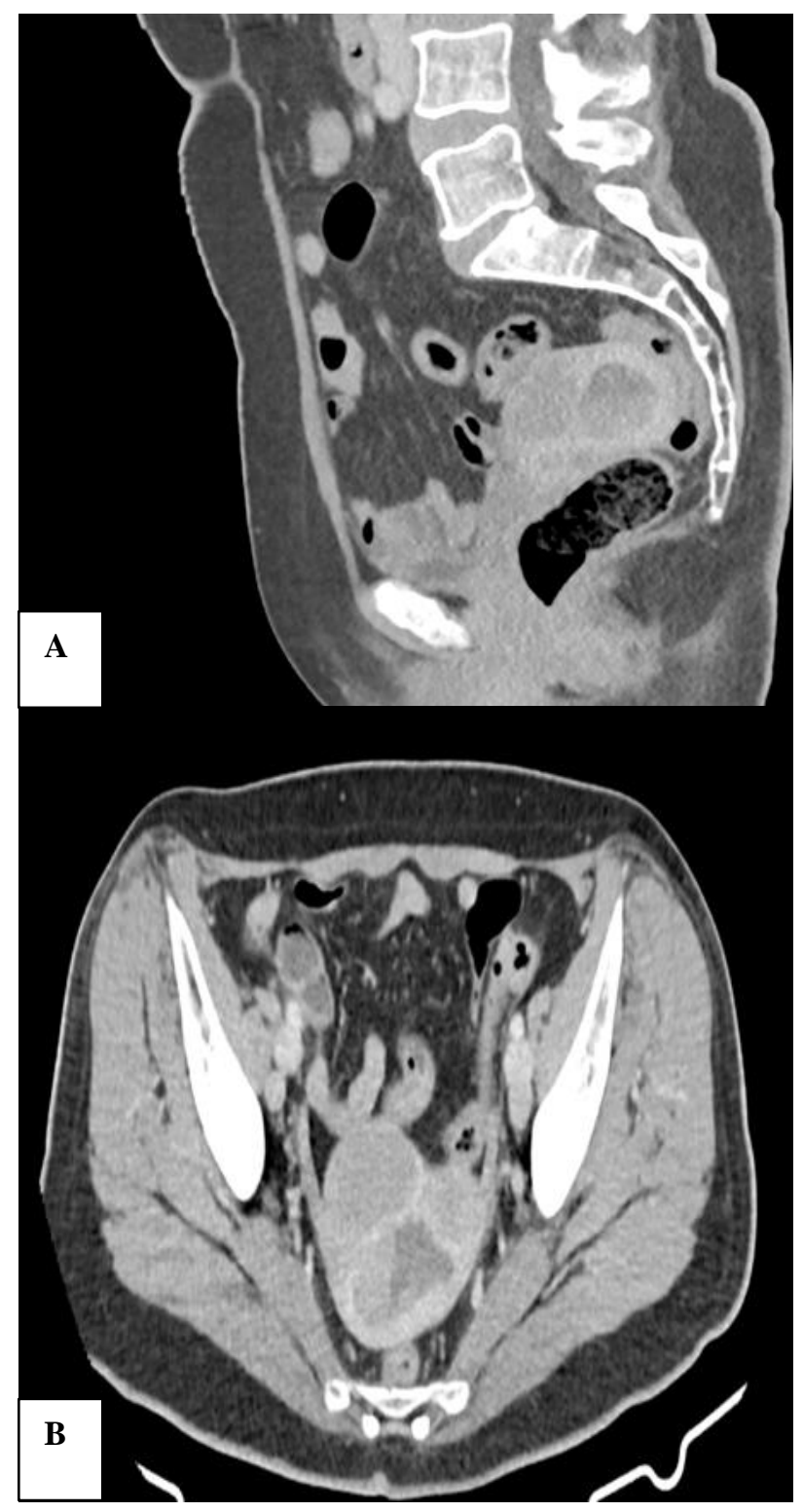

Figure 3: (A) staging thoracoabdominopelvic computed tomography-sagittal image; (B) staging thoracoabdominopelvic computed tomography axial image.

\section{DISCUSSION}

PGAD is a very perplexing entity since we are still trying to understand all the possible causes for it. ${ }^{3,7}$ There are no guidelines or algorithms for its diagnosis and treatment, so the published literature is particularly important in the guidance of the rare cases that physicians are confronted with. $^{2}$

In this case, the first difficulty was to understand the description of the symptoms by the patient, since she was woman with no coitarche or any type of sexual contact, so she first described the symptoms as discharges. After excluding urinary incontinence and asking about more sensations during the episodes we understood that maybe the patient was experiencing unwanted multiple orgasms, triggered by no apparent stimulus, and that were causing an elevated degree of distress, since she had no idea what was happening to her. This postmenopausal woman searched for help after some months having these symptoms and a diagnosis of PGAD was made. At that time, by excluding other possible aetiologies found in the literature, a psychological component was assumed, so an empirical pharmacological treatment with cloxazolam was started. ${ }^{2,7,9}$ Also, because of probable SGUM, local treatment with oestrogen and vaginal moisturizer was prescribed. Symptoms started to get better and after some months only very sporadic episodes were reported by the patient.

After three years of illness stabilization, another diagnosis was made - endometrial serous carcinoma at an advanced stage. She got appropriated surgery and is now under chemotherapy. Since the aetiology of PGAD is not completely known, although there was no description of this association in the literature, the possible aetiologies of PGAD were so diverse and still being discovere. ${ }^{3,7} \mathrm{We}$ sought this rare case should be disclosed. We are still trying to better understand the pathophysiology of PGAD and during the process of diagnosis, every physician goes to the literature and search for what is published. Our aim was to do a comprehensive review of PGAD and describe a rare case of a postmenopausal woman who developed this disease and during the follow-up was diagnosed with another serious disease which was advanced endometrial cancer. This reminds physicians to keep in mind the possibility of multiple diagnosis in the same patient, including the possibility of malignancy and the importance of maintained surveillance and high suspicion in clinical practice. The endometrial cancer was diagnosed in an advanced stage, despite annual gynaecological observation and we could not exclude that the assumed leiomyoma in the first ultrasound could already be the endometrial neoplasm. ${ }^{8}$

\section{CONCLUSION}

This case reported a diagnosis of PGAD, which is a very uncommon condition with aetiology and pathophysiology not completely known. We believe to have contributed by sharing this case with the medical community, alerting for the importance of PGAD diagnosis but also to essential ongoing follow-up of every women because other diagnosis may appear, as serious as malignancy.

\section{Funding: No funding sources \\ Conflict of interest: None declared \\ Ethical approval: Not required}

\section{REFERENCES}

1. Klifto KM, Dellon AL. Persistent genital arousal disorder: review of pertinent peripheral nerves. Sex Med Rev. 2020;8(2):265-73. 
2. Jackowich RA, Pink L, Gordon A, Pukall. CF. Persistent genital arousal disorder: A review of its conceptualizations, potential origins, impact, and treatment. Sex Med Rev. 2016;4(4):329-42.

3. Pink L, Rancourt V, Gordon A. Persistent genital arousal in women with pelvic and genital pain. J Obstet Gynaecol Can. 2014;36(4):324-30.

4. Leiblum SR, Nathan SG. Persistent sexual arousal syndrome: a newly discovered pattern of female sexuality. J Sex Marital Ther. 2001;27(4):365-80.

5. Parish SJ, Goldstein AT, Goldstein SW, Goldstein I, Pfaus J, Clayton AH, et al. Toward a more evidencebased nosology and nomenclature for female sexual dysfunctions-part II. J Sex Med. 2016;13(12):1888906.

6. McCabe MP, Sharlip ID, Atalla E, Balon R, Fisher AD, Laumann E, et al. Definitions of sexual dysfunctions in women and men: A consensus statement from the Fourth Consultation on Sexual Medicine 2015. J Sex Med. 2016;13(2):135-43.

7. Kruger THC. Can pharmacotherapy help persistent genital arousal disorder? Expert Opin Pharmacother. 2018;19(15):1705-9.

8. Waldinger MD, Venema PL, Gils APGV, Schweitzer DH. New insights into restless genital syndrome: static mechanical hyperesthesia and neuropathy of the nervus dorsalis clitoridis. J Sex Med. 2009;6(10):2778-87.

9. Facelle TM, Sadeghi-Nejad H, Goldmeier D. Persistent genital arousal disorder: characterization, etiology, and management. J Sex Med. 2013;10(2):439-50.

10. Amant F, Mirza MR, Koskas M, Creutzberg CL. Figo cancer report 2018: Cancer of the corpus uteri. Int J Gynecol Obstet. 2018;143(12):37-50.

Cite this article as: Sarabando R, Sousa N, Borges AC, Nogueira-Silva C. Persistent genital arousal disorder: always look beyond the surface: a case report. Int J Reprod Contracept Obstet Gynecol 2021;10:1691-4. 